\title{
Influencia del estado fenológico y nutrición de plantas matrices de café (Coffea arabica L.) en la
} producción de brotes, Rodríguez de Mendoza, Amazonas

Influence of the phenological state and nutrition of coffee matrix plants (Coffea arabica $L$.) on the production of sprouts, Rodríguez de Mendoza, Amazonas

Tito Sanchez Santillan ${ }^{1 *}$, Derlis Monsalve Goicochea ${ }^{2}$, Manuel Oliva $^{2}$, Luis Alberto Arévalo López

\section{RESUMEN}

El objetivo del experimento fue evaluar la influencia del estado fenológico y nutrición de plantas matrices de café (Coffea arabica L.) en la producción de brotes, Rodríguez de Mendoza, Amazonas. Se instaló bajo un diseño estadístico DBCA con arreglo factorial con factores: estado fenológico de plantas madres (producción, descanso) y tipo de fertilizantes (sin fertilizante, yaramila y molimax). Se tuvieron 6 tratamientos, 3 bloques y 18 unidades experimentales. En campo se seleccionaron e fueron sometidos a la inducción de brotes mediante la técnica de agobio, así mismo se aplicaron $40 \mathrm{~g}$ de cada fertilizante por planta dividido en dos aplicaciones 15 días antes y después del agobio. A los 120 días se evaluaron altura, diámetro, número y contenido de carbohidratos de brotes. Se encontró que los factores influyeron significativamente en la producción de brotes, mostrándose sobresaliente el estado fenológico descaso en el tamaño, diámetro y número de brotes. Por su parte, las plantas matrices fertilizadas con molimax y yaramila complex incrementaron la altura, diámetro y número. No obstante, obtuvo mayor número de brotes con alto carbohidrato con el tratamiento (producción + molimax café). Las plantas matrices de cafeto en estado fenológico descanso tienden a responder mejor en la producción de brotes y ésta se repotencia a medida que se encuentran mejor nutridas.

Palabras claves: altura, brote, fertilizantes, molimax, yaramila

\begin{abstract}
The objective of the experiment was to evaluate the influence of the phenological state and nutrition of coffee matrix plants (Coffea arabica L.) on the production of sprouts, Rodríguez de Mendoza, Amazonas. It was installed under a DBCA statistical design with factorial arrangement with factors: phenological state of mother plants (production, rest) and type of fertilizers (without fertilizer, yaramila and molimax). There were 6 treatments, 3 blocks and 18 experimental units. In the field, they were selected and subjected to shoot induction using the stress technique. Likewise, $40 \mathrm{~g}$ of each fertilizer per plant was applied, divided into two applications 15 days before and after stress. At 120 days height, diameter, number and carbohydrate content of shoots were evaluated. It was found that the factors significantly influenced the production of shoots, showing the outstanding phenological state in the size, diameter and number of shoots. For their part, the parent plants fertilized with molimax and yaramila complex increased their height, diameter and number. However, it obtained a higher number of shoots with high carbohydrate with the treatment (production + molimax coffee). Coffee plant matrices in a resting phenological state tend to respond better in the production of sprouts and this grows stronger as they become better nourished.
\end{abstract}

Keywords: height, sprout, fertilizers, molimax, yaramila

\footnotetext{
${ }^{1}$ Instituto de Investigaciones de La Amazonia Peruana (IIAP San Martín), Jirón Belén Torres de Tello N 135, Tarapoto, Perú

${ }^{2}$ Universidad Nacional Toribio Rodríguez de Mendoza de Amazonas (UNTRM-A), Instituto de Investigación para el Desarrollo Sustentable de Ceja de Selva, Calle Higos Urco N³42-350-356, Calle Universitaria N³04, Chachapoyas, Perú

"Autor de correspondencia. E-mail: titosanchezsantillan@gmail.com
} 


\section{INTRODUCCIÓN}

El Café es el primer producto agrícola peruano de exportación y es el séptimo país exportador de café a nivel mundial. No solo lidera las exportaciones agrícolas sino está dentro de los 10 principales productos de exportación, después de algunos minerales, petróleo, gas natural, harina de pescado, entre otros (MINAGRI, 2018). En el 2013, en el Perú se presentó un ataque masivo de roya que afectó las plantaciones de café especialmente a las variedades típica y caturra. El Ministerio de Agricultura peruano, ha reportado que hasta la fecha, la roya ha afectado el $50 \%$ de las 400 mil hectáreas de cafetales instalados a nivel nacional, lo que ha provocado una disminución del $20 \%$ en la producción de café, equivalente a una pérdida económica de S/.200 millones (Salazar y Rivera, 2013). En vista de que la roya acabo con la mayoría de las plantaciones de estas variedades, los agricultores han sembrado cafetales con variedades resistentes a la roya amarilla de café como el catimor. Esta variedad es excelente en producción pero bajo en calidad de taza por lo que sus quintales no son muy bien pagados (Julca-Otiniano et al.,2018). Esto contrata con variedades como las caturra y típica que tienen excelentes características físicas y de taza, y su comercialización es mejor pagada, además de ser plantas compacta con un buen potencial de rendimiento (Ramos et al., 2019).

Debido a la importancia económica y social del café para la región Amazonas, especialmente las variedades típica y caturra, se vio la necesidad de buscar estrategias para propagar y cultivar ambas variedades. A través de la propagación clonal de plantas élites de café, que superaron los daños provocados por la roya amarilla en las plantaciones (Obando et al., 2013). El proceso de producción de plantines enraizados se inicia con la obtención de plantas madre juveniles que han sido producidas en el laboratorio por embriogénesis somática y su establecimiento en jardines clonales. Después se usan camas hidropónicas donde las plantas madre se establecen a altas densidades para la producción periódica y abundante de rebrotes (Marques Cavalcanti Filho et al., 2018). Estudios preliminares de selección de plantas de café caturra que quedaron del ataque de roya, y que mostraron cierta tolerancia a ciertos hongo, evidenciaron la potencialidad que tiene esta estrategia de propagación. Sin embargo, es importante determinar si la nutrición y el estado fenológico de la planta afectara o no la calidad del brote producido (Barbon et al., 2014). Por estas razones, esta investigación tuvo como objetivo aportar nuevos conocimientos para futuras plantaciones clonales de café de la variedad caturra.

\section{MATERIALES Y MÉTODOS}

\section{Ubicación del experimento}

La investigación se ubicó en el distrito de Huambo en las coordenadas $6^{\circ} 20^{\prime} 10^{\prime \prime} \mathrm{s}, 77^{\circ} 27^{\prime} 58^{\prime \prime} \mathrm{o}$, a una altitud de 1630 m.s.n.m. en la provincia de Rodríguez de Mendoza. En la parcela de un productor en el caserío de Miraflores en el distrito de Huambo.

\section{Características del área experimental}

La parcela que se utilizó para esta investigación estuvo conformada por plantas de café de la variedad caturra de 7 a 8 años de edad, el terreno presentaba una pendiente del 10\%, con un tipo franco arcillo arenoso con un $\mathrm{pH}$ de 5,52 moderadamente ácido, un contenido medio de materia orgánica, bajo en fósforo y medio en potasio.

\section{Diseño estadístico del campo experimental}

La investigación fue de tipo experimental donde se trabajó con un diseño estadístico DBCA con arreglo factorial, con factores el primero estado fenológico $2 \mathrm{~A}$ y el segundo fue tipo de fertilizantes 3B, 6 tratamientos, 3 bloques y 18 plantas por cada unidad experimental (Tabla 1).

\section{Selección de plantas matrices de café caturra}

Para la selección del material experimental se determinaron los siguientes criterios:

Las plantas estaban en un período de producción y descanso, con edades de 7 a 8 años aproximadamente, se evaluó el tamaño y diámetro de tallos. Estos datos se registraron en una libreta de campo luego se pasó a un formato digital obteniendo un historial.

Así mismo, las plantas seleccionadas fueron codifica- 


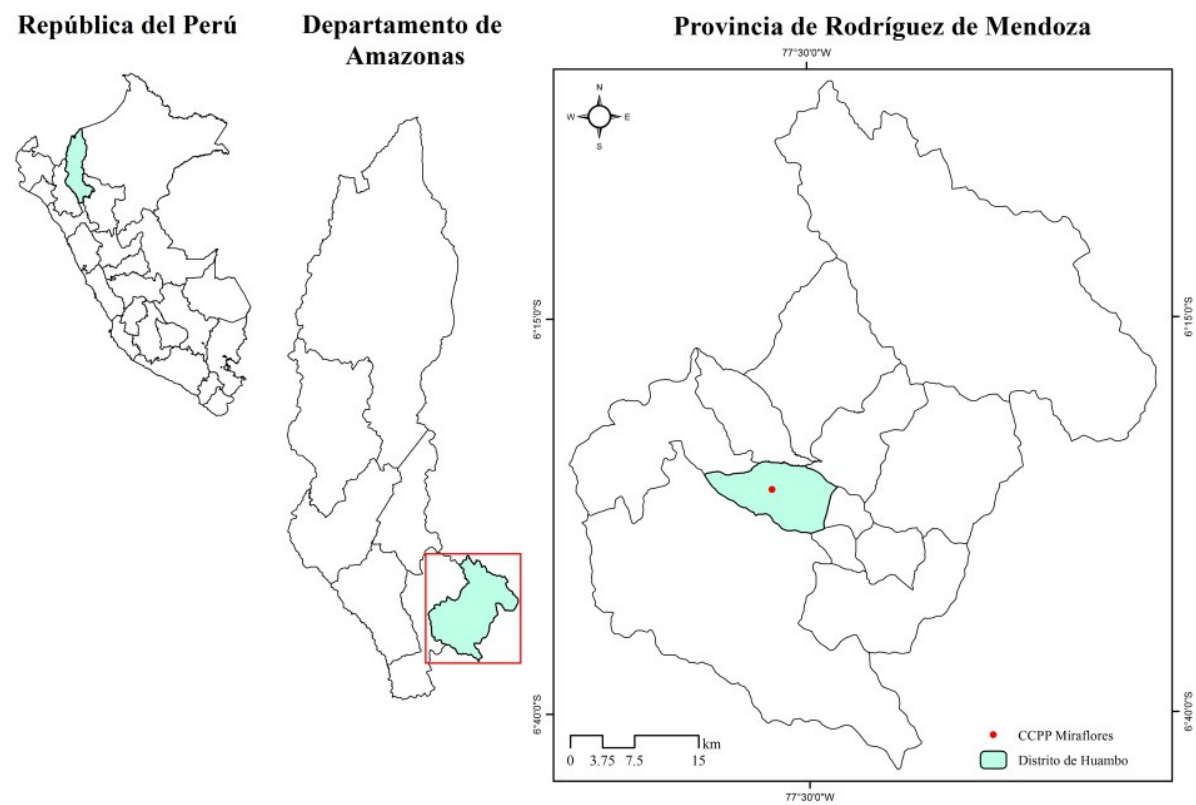

Figura 1. Ubicación geográfica del área de estudio en el caserío de Miraflores distrito Huambo (provincia de Rodríguez de Mendoza)

Tabla 1. Descripción de tratamientos de estudio

\begin{tabular}{|c|c|c|}
\hline Tratamiento & Código & Descripción \\
\hline T1 & a1b1 & $\begin{array}{l}\text { Producción }+ \\
\text { Sin fertilizante }\end{array}$ \\
\hline $\mathbf{T 2}$ & $\mathrm{a} 1 \mathrm{~b} 2$ & $\begin{array}{l}\text { Producción }+ \\
\text { Molimax café }\end{array}$ \\
\hline T3 & a1b3 & $\begin{array}{l}\text { Producción }+ \\
\text { Yaramila } \\
\text { complex }\end{array}$ \\
\hline T4 & $\mathrm{a} 2 \mathrm{~b} 1$ & $\begin{array}{c}\text { Descanso }+ \text { Sin } \\
\text { fertilizante }\end{array}$ \\
\hline T5 & $\mathrm{a} 2 \mathrm{~b} 2$ & $\begin{array}{l}\text { Descanso+ } \\
\text { Molimax café }\end{array}$ \\
\hline T6 & $a 2 b 3$ & $\begin{array}{c}\text { Descanso }+ \\
\text { Yaramila } \\
\text { complex }\end{array}$ \\
\hline
\end{tabular}

das adecuadamente, registrando el número de planta, tratamiento y bloques; Se seleccionaron las plantaciones de acuerdo al grosor del tallo, de 25 a $35 \mathrm{~mm}$ de diámetro y la medición del grosor del tallo se realizó a la altura de la cintura, al finalizar, se etiquetaron una a una las plantas seleccionadas.

\section{Aplicación de la primera fertilización a plantas matrices de café}

La aplicación del fertilizante se realizó al segundo día después del etiquetado con la aplicación del $50 \%$ de la cantidad requerida según el análisis de suelo. Los fertilizantes comerciales utilizados fueron Molimax café, cuya composición química es de $20 \%$ nitrógeno
(N), 7\% fósforo $\left(\mathrm{P}_{2} \mathrm{O}_{5}\right), 20 \%$ potasio $\left(\mathrm{K}_{2} \mathrm{O}\right), 3 \%$ magnesio $(\mathrm{MgO})$ y $4 \%$ azufre $(\mathrm{S})$ y yaramila complex, con la siguiente composición química: $12 \% \mathrm{~N}, 5 \%$ nitrato, $11 \%$ fósforo $(\mathrm{P} 2 \mathrm{O} 5), 18 \%$ potasio $\left(\mathrm{K}_{2} \mathrm{O}\right), 2,7 \%$ magnesio $(\mathrm{MgO}), 8 \%$ óxido de azufre $\left(\mathrm{SO}_{3}\right), 0,015 \%$ boro (B), $0,2 \%$ fierro (Fe), $0,02 \%$ manganeso $(\mathrm{Mn})$ y $0,02 \%$ $\operatorname{zinc}(\mathrm{Zn})$.

Debido a la pendiente del terreno, los fertilizantes fueron aplicados a media luna en la parte superior de la planta, a una profundidad de 5 a $7 \mathrm{~cm}$ formando la banda de aplicación, utilizándose un rastrillo para evitar daños en los pelos absorbentes, los fertilizantes se aplicaron en la banda de aplicación y se taparon con la tierra usando el rastrillo (Castañeda, 2000).

\section{Agobio e inducción de plantas matrices de café}

Después de los 15 días de la primera fertilización, se cortaron las ramas hasta el tercio medio del tamaño de planta con ayuda de una tijera podadora; luego éstas fueron "agobiadas o inclinadas" con un ángulo de 45 grados respecto al suelo, siendo sujetadas con una cinta rafia a una estaca. La dirección de las plantas agobiadas estuvo en sentido de este a oeste, para que toda la planta estuviera expuesta a la radiación solar y así favorecer la inducción de brotes. Este primer procedimiento permite que la planta asimile los nutrientes y se prepare para la segunda fertilización.

76 Rev. de investig. agroproducción sustentable 3(1): 74-82, 2019 ISSN: 2520-9760 


\section{Segunda aplicación de fertilizantes a las plantas de café}

La segunda aplicación se realizó a los 15 días después de haber realizado el agobio aplicando el 50\% de los fertilizantes restantes, este criterio se tomó en cuenta para nutrir las plantas antes y después de ser agobiadas, y el otro $50 \%$ se aplicaría con la finalidad de que ayude a la emisión y nutrición de los brotes.

\section{Evaluación de las variables}

Las evaluaciones se realizaron seleccionando 3 brotes por planta: un brote del tercio inferior, otro del tercio medio y otro de tercio superior.

- Altura de brote: Ésta variable se evaluó durante 4 meses, donde se realizaron 6 evaluaciones cada 15 días, a partir de $10 \mathrm{~mm}$ como mínimo de altura del brote. Las medidas se tomaron desde la base hasta la parte apical del brote utilizando una regla milimetrada. Se evaluaron 3 brotes por planta los cuales fueron codificados para siempre evaluar el mismo brote.

- Diámetro de brote: Se evaluó con ayuda de un vernier digital milimetrado, en el momento de evaluar la altura de la planta se tomaron los datos a $10 \mathrm{~mm}$ de altura de la base.

- Número de brotes: Al final de los 4 meses se contabilizaron el número total de brotes producidos por la planta madre como efecto del estado fenológico y nutrición.

- Nivel de carbohidratos: Para determinar la presencia de almidón deseable se llevó a laboratorio 3 brotes e café por planta y se utilizó la prueba del yodo. Éste método consiste en sujetar los extremos recién cortados de un manojo de estacas y sumergirlas por un minuto en una solución de $0.02 \%$ de yoduro de potasio. Las estacas con mayor contenido de almidón se tiñeron de un color más oscuro. Esto permitió hacer una clasificación de las estacas ricas, medianas y pobres en carbohidratos (Lopes et al., 2016)

\section{RESULTADOS Y DISCUSIÓN}

El análisis de varianza (ANOVA), establece que existe alta diferencia significativa entre los estados fenológicos para la altura y número de brotes, y existe una diferencia mínima significativa para el diámetro de brotes. El tipo de sustratos muestran alta diferencia significativa para la altura, diámetro y número de brotes. Del mismo modo se observó que los factores influyeron significativamente de manera interaccionada, mostrando que son complementarios entre cada factor (p-valor $<0,05)$ (Tabla 2).

Tabla 2. Valores de probabilidad (p-valor $<0,05)$ en el análisis de varianza en la producción de brotes de café (Coffea arabica L.) variedad caturra

\begin{tabular}{cccc}
\hline Fuente de Variación & Altura brote $\mathbf{( c m )}$ & Diámetro brote $(\mathbf{c m})$ & Número brotes \\
\hline Estado Fenológico & $0,0000^{*}$ & $0,0466^{*}$ & $0,0000^{*}$ \\
Tipo de fertilizante & $0,0000^{*}$ & $0,0000^{*}$ & $0,0004^{*}$ \\
Estado Fenológico*Tipo de fertilizante & $0,0000^{*}$ & $0,0000^{*}$ & $0,0049^{*}$ \\
\hline
\end{tabular}

*=significancia

Tabla 3. Comparación múltiple según el test de Tukey $(\alpha=0.05)$ para la producción de brotes de café (Coffea arabica L.) variedad caturra

\begin{tabular}{cccc}
\hline Fuente de variación & Altura brote $\mathbf{( c m )}$ & Diámetro brote (cm) & Númerobrotes \\
\hline Estado fenológico & & & \\
\hline Descanso & $23,90 \mathrm{a}$ & $6,38 \mathrm{a}$ & $37,43 \mathrm{a}$ \\
Producción & $21,26 \mathrm{~b}$ & $6,13 \mathrm{~b}$ & $29,57 \mathrm{~b}$ \\
\hline Fertilizante & & & $28,47 \mathrm{~b}$ \\
Sinfertilizante & $20,09 \mathrm{c}$ & $5,68 \mathrm{~b}$ & $37,56 \mathrm{a}$ \\
Yaramila complex & $22,07 \mathrm{~b}$ & $6,49 \mathrm{a}$ & $34,47 \mathrm{a}$ \\
Molimax café & $25,57 \mathrm{a}$ & $6,61 \mathrm{a}$ & \\
\hline
\end{tabular}


En la tabla 3, se observa según el test de Tukey $(\alpha=0.05)$, que existe diferencia significativa influenciados por estado fenológico, sobresaliendo el estado descanso en la altura $(23.9 \mathrm{~cm})$, diámetro $(6.38 \mathrm{~mm})$ y número de brotes (37.43). Así mismo dentro del tipo de fertilizante, aplicaciones de molimax y yaramila favorecieron los incrementos de altura, diámetro y número de brotes, mostrándose ambos superiores respecto al testigo ( $\sin$ fertilizante).
En la figura 2, se observa el nivel de carbohidratos influenciados por el estado fenológico y tipo de fertilizante de plantas madres, donde el estado de producción y fertilizados con molimax café, fueron superiores en presentar alto carbohidrato. Sin embargo, el estado tanto descanso y producción $+\sin$ fertilizante presentaron pocos brotes con nivel alto de carbohidratos, denotando que la fertilización es un factor determinante en la producción de carbohidratos.

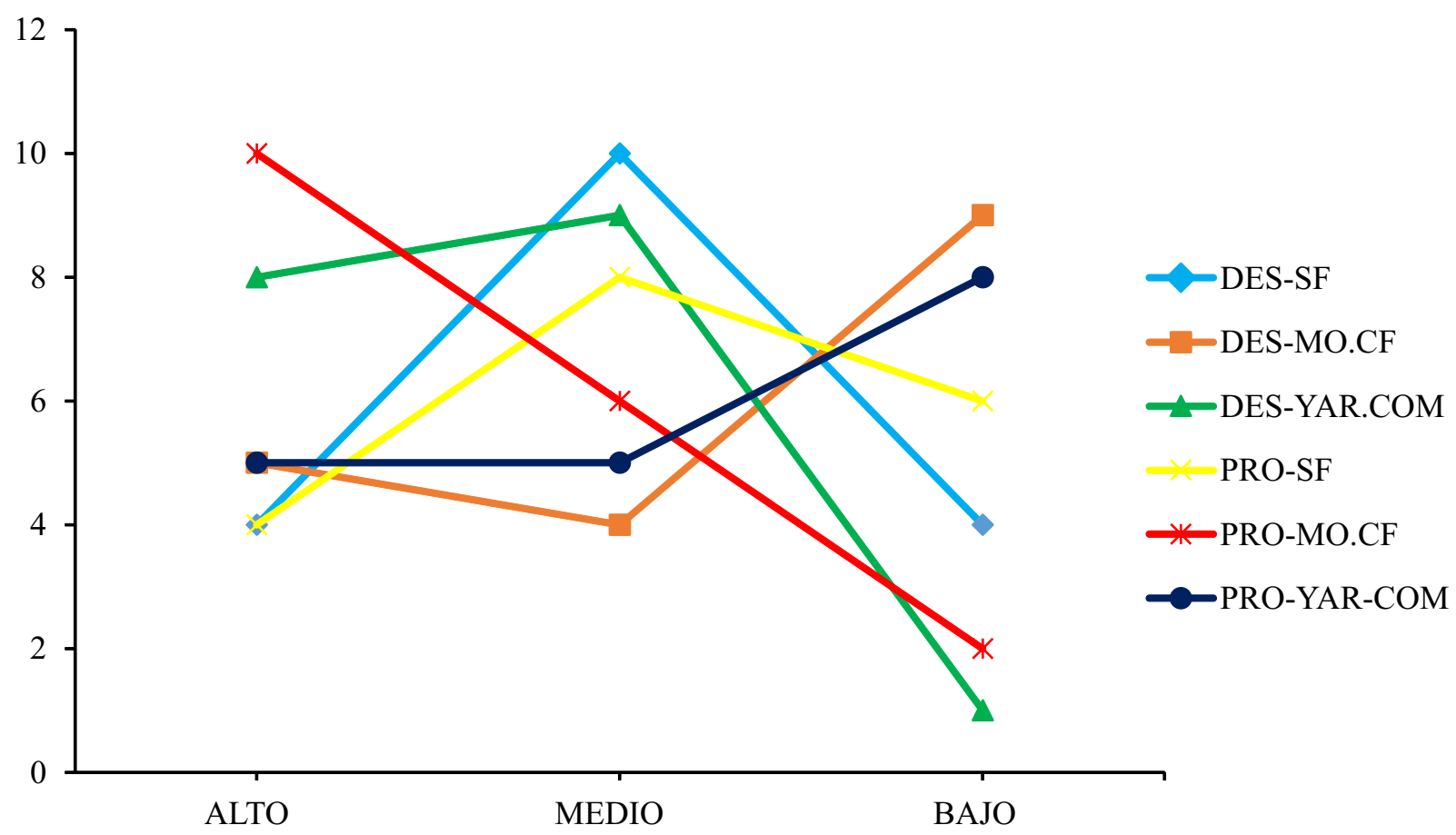

Figura 2. Nivel de carbohidratos según el estado fenológico y tipo de fertilizante en brotes de Coffea arabica L. variedad caturra. $\mathrm{DES}=$ descanso; $\mathrm{SF}=$ sin fertilizante; $\mathrm{MO}-\mathrm{CF}=$ molimax café; YAR.COM=yaramila complex; $\mathrm{PRO}=$ producción.

Los factores en estudio tuvieron efectos simples y la interacción entre ellos, altamente significativos estadísticamente; sobre los parámetros de tamaño de brote, número de brotes y diámetro de brotes. El tamaño del brote es uno de los indicadores de calidad de la planta, por lo que es la variable más visible para este tipo de análisis. El tamaño, estuvo fuertemente influenciado por el estado fenológico de descanso, donde se reportaron medias de $23,9 \mathrm{~cm}$, comparado con el estado fenológico de producción, presentando este último una media de $21,26 \mathrm{~cm}$. Esto se debe a que las plantas madres deben mostrar un crecimiento vegetativo activo (no deben haber entrado en floración) para que tengan la más alta capacidad generativa (Parlak and Semizer-Cuming 2012). No obstante, esto puede variar según las condiciones ambientales y el manejo que se le esté dando al cultivo (Meléndez and Molina 2002).

El fertilizante 20N-7P-20K molimax café, mostró mejores efectos respecto al fertilizante Yaramila complex, indicando que el tipo de fertilizante puede influir de manera positiva en el tamaño de brotes del cafeto. Así mismo se observó que las plantas matrices sin fertilizante tuvieron el menor desarrollo que aquellas fertilizadas. El nitrógeno es el principal nutriente para el crecimiento y desarrollo de las plantas, el cual es absorbido por las raíces preferentemente en forma de nitrato (NO3) o amonio (NH4+). Las plantas que fueron fertilizadas con $20 \mathrm{~N}-$ 7P-20K molimax café, obtuvieron brotes de mayor 
tamaño debido a que tiene en su composición $20 \%$ de nitrógeno, es decir tiene $8 \%$ más de $\mathrm{N}$ que el 12N-11P$18 \mathrm{~K}$ yaramila complex. Por otro lado, el $12 \mathrm{~N}-11 \mathrm{P}-$ $18 \mathrm{~K}$ yaramila complex en su composición contiene zinc que juega un papel importante en la biosíntesis de las auxinas (Las auxinas son un grupo de fitohormonas) que funcionan como reguladoras del crecimiento vegetal (Ac. Indolacético), el zinc participa en el metabolismo vegetal como activador de varios enzimas y posiblemente haya regulado el crecimiento de los brotes. Principalmente a que en la fertilización en etapa de producción debe contener altas dosis de nutrientes, con el fin de garantizar los requerimientos que demanda el cultivo en cada una de sus fases, especialmente en la de producción; lo cual estaría asociado con el uso del molimax café, por su mayor contenido de N y K (Sadeghian and GonzálezOsorio 2012).

Asimismo, la Federación Nacional de Cafetaleros de Colombia, menciona que la etapa de almácigo, es donde la planta responde de manera positiva a abonos orgánicos y especialmente a las concentraciones de fósforo, más que a las concentraciones de nitrógeno y potasio, en términos de altura. Mientras que, en la etapa de crecimiento vegetativo, los requerimientos nutricionales de nitrógeno, son mayores, seguidos del fósforo y potasio, los cuales deben ser incrementados, al iniciar la etapa reproductiva (Sadeghian 2008). Como lo demuestran los resultados, donde el estado fenológico de descanso asimiló mucho mejor la concentración de nutrientes aportadas por el fertilizante.

La variable diámetro es un indicador morfológico y se asocia con el vigor y la resistencia mecánica de la planta, por lo que entre más robusta este, más posibilidades de sobrevivir (Gardiner et al.,2009). De modo que, si una planta tiene los requerimientos nutricionales suficientes para su desarrollo, será más robusta y sana (Sigala et al.,2012). El diámetro de los brotes fue directamente proporcional al tamaño; observándose que tanto el factor fenológico descanso y el tipo de fertilizante juega un papel importante en la tasa de crecimiento, influyendo significativamente en los procesos. Se encontró que yaramila complex, mas estado fenológico descanso, de la planta matriz presentó mayor número de brotes $(45,35)$, siendo éste, el mejor tratamiento comparado con los demás. Del mismo modo se encontró que las plantas matrices de café en los dos estados fenológicos (producción y descanso) sin la aplicación de algún tipo de fertilizante generaron menor número de brotes; el número de brotes tiene una fuerte asociación con la eficiencia fotosintética de la planta y su capacidad para absorber nitrógeno (Sanclemente \& Peña, 2008), por lo que este nutriente llega a ser el limitante en la productividad de las plantas (Corrales-González, Rada, and Jaimez 2016). Las plantas élites que tuvieron menor número de brote fueron las que estaban sin fertilizante. Sin embargo, el mayor número de brotes se presentó con el fertilizante yaramila Complex que tiene un porcentaje menor de nitrógeno $(12 \%)$ a diferencia del fertilizante molimax café (20\%). Probablemente, este efecto estuvo asociado al aporte de los micronutrientes del fertilizante yaramila complex, comparado con el molimax Café. No obstante, en la tabla 2 se observa que las plantas élite estaban en la etapa fenológica de descanso lo cual tuvo alta diferencia significativa respecto a producción. El estado fenológico de descanso, es donde la planta presenta un crecimiento vegetativo activo teniendo la más alta capacidad generativa y de producción. El factor estado fenológico, tiene un efecto significativo en el número de brotes. Cuando las plantas madres son cultivadas en condiciones de deficiencia de fósforo, potasio, magnesio y calcio, la formación de raíces en las estacas obtenidas de ellas, no tienen las mejores condiciones a diferencia de las plantas con nutrición completa. Sin embargo, con la reducción de nitrógeno en las plantas madre se aumentaba la formación de raíces en las estacas. Posteriormente, se observó, que la deficiencia extrema de nitrógeno en las plantas madre, reduce en vez de aumentar el enraizado (DaMatta et al.,2007).

La concentración de carbohidratos puede variar según 
las condiciones del ambiente y las fases fenológicas de la planta, siendo un producto fundamental de la fotosíntesis, mejorando su vitalidad y habilidad para tolerar el estrés (Martínez-Trinidad, PlascenciaEscalante, and Islas-Rodríguez 2013). El más alto nivel de carbohidratos se presentó en los brotes obtenidos en la fase de producción y con el fertilizante molimax café T2 (nivel alto 10, nivel medio 6 y nivel bajo 2 brotes). Esto se debe a que molimax café tiene un $20 \%$ de potasio, 2\% más que yaramila, según (Programa de Desarrollo Alternativo en Satipo, 2017) el $\mathrm{K}$ activa la enzima que regula síntesis de almidones, su deficiencia reduce sus niveles, eso también nos indica los buenos resultados obtenidos con el tratamiento 6 (nivel alto 8 , nivel medio 9 y nivel bajo 1 brotes). Por consiguiente, los carbohidratos, son responsables de la formación de los órganos vegetativos de las plantas, proporcionando la energía necesaria para el nacimiento de los brotes de las especies perennes o anuales (Arcila-Pulgarín 2007).

El crecimiento secundario de las especies perennes representa una alta demanda de minerales, necesarios para la actividad del cambium. Asimismo, la etapa de almácigo, es donde la planta responde de manera positiva a abonos orgánicos y especialmente a las concentraciones de fósforo, más que a las concentraciones de nitrógeno y potasio en términos de altura (Meléndez and Molina 2002). En la etapa de crecimiento vegetativo, los requerimientos nutricionales de nitrógeno, son mayores, seguidos del fósforo y potasio, los cuales deben ser incrementados, al iniciar la etapa reproductiva (Sadeghian 2008)-

Finalmente, es importante mencionar que aunque la reproducción vía sexual no es el único modo de reproducción del cafeto, se puede acudir a otras estrategias de propagación asexual usando con frecuencia el injerto y la estaca (Vásquez-Inuma et al.,2018). Por lo que se infiera que, el cultivo por brotes, es una muy buena alternativa, ya que, permite que las plantas de café presenten características específicas que aporten una mayor producción y calidad de las diferentes plantaciones del café en la zona de estudio (Ono, Rodrigues, and de Pinho 1992), previniendo la contaminación de hongos como la roya. Adicional a esto, estás estrategias de cultivo, permiten producir plantas en menor tiempo, con mayor capacidad para soportar el estrés (Arizaleta and Pire 2008),

\section{CONCLUSIONES}

Los brotes de café mostraron incrementos en altura, diámetro y número, influenciados por el estado fenológico descanso. Así mismo los fertilizantes molimax café y yaramila complex mostraron efectos positivos en la producción de brotes, mostrando superioridad respecto al testigo ( $\sin$ fertilizante). Estado fenológico producción + molimax café, generaron mayor brotes con alto nivel de carbohidratos.

Se demostró que las plantas matrices de cafeto en estado fenológico descanso tienden a responder mejor en la producción de brotes y ésta se repotencia a medida que se encuentran mejor nutridas.

\section{REFERENCIAS BIBLIOGRÁFICAS}

Arcila-Pulgarín, J. 2007. “Capitulo 2. Crecimiento y Desarrollo de La Planta de Café." En Sistemas de Producción de Café En Colombia, Ospina, H. F., S. Milena-Marín. Chinchina, Colombia: Ceincafe.

Arizaleta, M., y Reinaldo P. 2008. "Respuesta de Plántulas de Cafeto Al Tamaño de La Bolsa y Fertilización Con Nitrógeno y Fósforo En Vivero." Agrociencia 42 (1): 47-55.

Barbon, R., H. N. Thi, A. Capote, M. De Feria, A. Pérez, L. Rivero, M. Leiva, y O. Hurtado. 2014. "Efecto de Mezclas de Sustratos En La Fase de Conversión de Plantas de Coffea arabica L. Cv. 'Caturra Rojo' Obtenidas Por Embriogénesis Somática." Biotecnología Vegetal 14(4): 205-13.

Castañeda, E. 2000. ABC Del Café: Cultivando Calidad. Lima (Perú): Bekos S.A.

Corrales-González, M., F. Rada, y R. Jaimez. 2016. "Efecto Del Nitrógeno En Los Parámetros 
Fotosintéticos y de Producción Del Cultivo de La Gerbera (Gerbera jamesonii H. Bolus Ex Hook. F.)." Acta Agronomica 65 (3). DOI: 10.15446/acag.v65n3.49555.

DaMatta, F. M., C. P. Ronchi, M. Maestri, y R. S. Barros. 2007. "Ecophysiology of Coffee Growth and Production.” Brazilian Journal of Plant Physiology 19 (4): 485-510.

Gardiner, E. S., D. F. Jacobs, R. P. Overton, y G. Hernandez. 2009. "Root-Collar Diameter and Third-Year Survival of Three Bottomland Hardwoods Planted on Former Agricultural Fields in the Lower Mississippi Alluvial Valley." In National Proceedings: Forest and Conservation Nursery Associations. Dumroese R. K., L. E. Riley (eds). Fort Collins (EEUU): Department of Agriculture, Forest Service, Rocky Mountain Research Station.

Julca-Otiniano, A., G. Alarcón-Águila, L. AlvaradoHuamán, R. Borjas-Ventura, y V. CastroCepero. 2018. "Behavior of Three Cultivars of Coffee (Catimor, Colombia and Costa Rica 95) in the Valley of El Perené, Junín, Perú.”

Chilean Journal of Agricultural and Animal Sciences 34 (3): 205-15. DOI: 10.4067/S0719-38902018005000504.

Lopes, Guido R., Andreia S. Ferreira, Mariana Pinto, Cláudia P. Passos, Elisabete Coelho, Carla Rodrigues, Cláudia Figueira, Sílvia M. Rocha, Fernando M. Nunes, and Manuel A. Coimbra. 2016. "Carbohydrate Content, Dietary Fibre and Melanoidins: Composition of Espresso from Single-Dose Coffee Capsules." Food Research International 89: $\begin{array}{llllll}9 & 8 & 9 & - & 9 & 6\end{array}$. https://doi.org/10.1016/j.foodres.2016.01.01 8.

Marques Cavalcanti Filho, P. F., D. Corona Baitelle, S. de Jesus Freitas, W. Souza da Silva, P. C. dos Santos, W. Pereira Rodrigues, S. de Paiva Freitas, et al.,2018. "Effect of Growth
Regulators in Production and Rooting of Coffea arabica L. Minicuttings." American Journal of Plant Sciences 9 (04): 628-36. DOI: 10.4236/ajps.2018.94049.

Martínez-Trinidad, T., F. O. Plascencia-Escalante, y L. Islas-Rodríguez. 2013. "Relationship Between Carbohydrates and Vitality in Urban Trees." Revista Chapingo Serie Ciencias Forestales y Del Ambiente XIX (3): 459-68. DOI: 10.5154/r.rchscfa.2012.03.016.

Meléndez, G., y E. Molina. 2002. Fertilización Foliar: Principios y Aplicaciones. San José (Costa Rica): Centro de Investigaciones Agronómicas. Universidad de Costa Rica.

MINAGRI. 2018. Situación Actual Del Café En El Pais. http://minagri.gob.pe/portal/485-feriascaa/10775-el-cafe-peruano (Consultada el 18 de abril de 2018).

Obando, N. V., C. Mestanza, y M. Oliva. 2013. “Efecto Del Manejo Cultural y Caldo Bordelés Sobre La Roya Del Café (Hemileia vastatrix) En La Provincia de Rodríguez de Mendoza, Amazonas." Revista INDES 1 (2): 51-58. DOI: $10.25127 /$ indes.201302.006.

Ono, E. O., J. D. Rodrigues, y S. Z. de Pinho. 1992. "Estudio de La Influencia Del Tiempo de Recolección de Las Ramas, En El Enraizamiento Del Tallo Del Café (Coffea arabica L. Cv 'Mundo Novo')." Scientia Agricola 49 (1): 29-35.

Parlak, S., y D. Semizer-Cuming. 2012. “Anatomical Examination of Root Formation on Bay Laurel (Laurus Nobilis L.) Cuttings.” Journal of Plant Biology Research 1 (4): 145-50.

Ramos, E., I. Lima-Medina, y G. B. Cornejo-Condori. 2019 . "Comparativo de Calidad Organoléptica de Café (Coffea arabica L.) En Puno - Perú y La Paz - Bolivia.” Journal of High Andean Research 21 (2): 283-92. DOI: 10.18271/ria.2019.505 REV.

Sadeghian, S. 2008. "Fertilizad Del Suelo y Nutricion Del Cafe En Colombia." Cenicafé (32): 1-54. 
Sadeghian, S., y H. González-Osorio. 2012.

"Alternativas Generales de Fertilización

Para Cafetales En La Etapa de Producción.”

Cenicafé (424): 1-8.

Salazar, B., y N. Rivera. 2013. "La roya: devastacion del sector cafetalero." La Revista Agraria (151): 1-14.

Sigala, J., G. Sosa, M. Martinez-Salvador, y R. J. Soto. 2012. Influencia de La Calidad de Planta En La Supervivencia y Crecimineto de Plantaciones Forestales En Chihuahua. Chihuahua, México: SAGARPA, INIFAP.

Vásquez-Inuma, L. L., D. Ayala-Montejo, G. VallejosTorres, L. Arévalo-López, C. BustamanteOchoa, E. Calixto-Vásquez, y E. RamosVásquez. 2018. "Edad Del Material Vegetativo y Su Efecto En El Enraizamiento de Brotes de Café (Coffea arabica) Variedad Caturra." Investigación Valdizana 12 (4): 215-26. DOI: 10.33554/riv.12.4.157. 